\title{
Psychosocial Status and Mental Health in Adolescents before and after Bariatric Surgery: A Systematic Literature Review
}

\author{
Sabine Herget Almut Rudolph Anja Hilbert Susann Blüher \\ Integrated Research and Treatment Center (IFB) Adiposity Diseases, University of Leipzig, \\ Leipzig, Germany
}

\section{Key Words}

Adolescent bariatric surgery · Pre-operative mental health · Post-operative mental health .

Depressive disorders · Eating disorders

\begin{abstract}
Objective: As long-term results of conservative treatment for obesity are discouraging, bariatric surgery is becoming a treatment option for extremely obese adolescents. However, mental and behavioral problems need to be respected when treating this vulnerable target group. Methods: A detailed systematic literature review on pre- and post-operative depressive, anxiety and eating disorder symptoms of adolescent patients was performed in PsychINFO, PubMed and Medline electronic databases. Results: Twelve studies met the inclusion criteria. Although strength of evidence was limited, results suggested that pre-operatively a third of adolescents suffered from moderate to severe depressive disorder symptoms and a quarter from anxiety disorder symptoms, while a substantial number showed eating disorder symptoms. Post-operatively, levels of depressive disorder symptoms significantly improved. Original articles on outcomes of eating and anxiety disorder symptoms after weight loss surgery were not found. Conclusions: Further attention is needed on consistent clinical assessment of mental health disturbances and their consecutive treatment in adolescents. Future research should also focus on psychological and psychosocial predictors of weight loss after bariatric surgery.


Herget et al.: Psychosocial Status and Mental Health in Adolescents before and after Bariatric Surgery: A Systematic Literature Review

\section{Introduction}

Obesity is one of the most serious global public health problems in the 21st century. Currently more than 1.4 billion adults are obese, and already 40 million children suffer from overweight and obesity worldwide [1]. Among children and adolescents, overweight and obesity are defined based on nationally representative data sets and expressed in percentiles. According to the International Obesity Task Force (IOTF), overweight in childhood and adolescence is classified as a BMI value between the 85th and the 95th percentile, and obesity is defined by BMI values above the 95th percentile $[2,3]$. To date, overweight and obesity prevalence among children and adolescents is stabilizing, albeit at an alarmingly high level $[4,5]$.

Obesity correlates with a plethora of comorbidities which range from serious metabolic and cardiovascular disorders, severe sleep apnea or hypertension to psychiatric problems such as depression, anxiety and a low quality of life [6, 7]. Lifestyle interventions, which are termed as the conservative treatment for overweight and obesity, have shown limited longterm efficacy for severely obese adolescents [8-11]. Therefore, bariatric surgery is increasingly regarded as a suitable treatment option for severely obese adolescents, in whom conservative weight loss treatment approaches have failed [12].

Bariatric surgery has been established as the most successful and cost-effective treatment for adult patients with severe obesity that significantly minimizes metabolic comorbidities such as type 2 diabetes and maximizes long-term weight loss [13, 14]. Bariatric surgery procedures are either based on a restriction of the capacity of the stomach or on the creation of a malabsorptive environment. Four procedures are typical, comprising two restrictive methods (i.e., laparoscopic adjustable gastric banding (LAGB) and laparoscopic sleeve gastrectomy (LSG)), and two combined restrictive-malabsorptive methods (biliopancreatic diversion (BPD), Roux-en-Y gastric bypass (RYGB)) [15]. To date, only limited data are available on bariatric surgery in adolescent patients, but significant post-operative weight loss and remarkable improvements of metabolic and cardiovascular comorbidities have been reported by several groups [16-20].

However, all procedures imply specific medical and psychological problems which may even increase in adolescent patients due to longer post-operative life expectancy compared to adult patients [21]. From a psychosocial point of view, adolescent bariatric surgery candidates represent an especially vulnerable group of patients as they are still in a phase of development of self-concept, influenced by peer relationships and their social and educational environment [22, 23]. Empirically, psychopathology of obese adolescents includes externalizing and internalizing behavioral problems, depressive, anxiety and eating disorder symptoms [24]. Obese adolescents more often suffer from body dissatisfaction, anhedonia, low self-esteem, and depressive disorder symptoms than their normal-weight peers [25]. Studies on adult bariatric surgery candidates have demonstrated conflicting results on association between pre-surgery psychopathology and post-operative outcome. Some studies report no psychological or behavioral predictors of weight loss [26, 27]. Others, however, document that pre-operative psychopathology can interfere with post-operative outcomes, suggesting the need for an extensive pre-operative psychosocial assessment and treatment [28-30]. It is absolutely necessary to further describe the mental health status of adolescents who consider bariatric surgery procedure in order to offer appropriate patient education and establish suitable medical and psychological pre- and post-operative guidelines. A protocol for a comprehensive mental health assessment for adolescent bariatric surgery candidates has recently been published, briefly indicating prevalence rates of mental health disorders, but pointing to the need for more emphasis on this critical topic [31]. Furthermore, a recent review by Kubik et al. [32] briefly touched the topic regarding the impact of bariatric surgery 
Herget et al.: Psychosocial Status and Mental Health in Adolescents before and after

Bariatric Surgery: A Systematic Literature Review

in childhood, but did not focus in detail on severity, prevalence and outcomes of mental health disorders in these patients.

Therefore, the objective of this paper was to conduct a detailed systematic review of the literature concerning psychosocial status and mental health in adolescent bariatric surgery patients. Because a significant number of adolescent bariatric surgery candidates reported mental and general health problems [33], this review focuses on prevalence rates of common pre- and post-operative mental health problems (i.e., depressive, anxiety and eating disorder symptoms) in adolescent patients, who are presenting for being accepted into a bariatric surgery program.

\section{Material and Methods}

Relevant articles were identified through search of PsychINFO, PubMed and Medline electronic databases including combinations of the following search terms: weight loss surgery, surgical weight loss, bariatric surgery, gastric bypass, gastric banding plus adolescents, adolescence, youth plus psychosocial, psychological, mental health, behavioral disorders or depressive disorder, anxiety disorder, eating disorders. Furthermore, relevant papers were extracted from reference lists of retrieved articles, and pertinent journals were searched by hand to identify additional studies.

Inclusion criteria were as follows: studies published in English language in peer-reviewed journals prior to February 2014 examining the psychosocial status and mental health of adolescent patients before and after bariatric surgery. Thus, observational studies and scientific reports were included when they assessed mental health and psychosocial outcome variables in adolescent patients (age 12.5 to <18 years) before and/ or after bariatric surgery. Discussion papers, reviews, and comments were excluded. Authors removed duplicates among the identified studies, independently screened titles and abstracts for relevance, and if necessary reviewed full-text for inclusion and exclusion criteria. Subsequently, information on mental health in adolescent patients before and after bariatric surgery were extracted and tabulated.

\section{Results}

\section{Description and Content of Included Studies}

After removal of duplicates and exclusions, a total of 12 studies that assessed psychosocial status and mental health before and after bariatric surgery and that met all criteria for inclusion in our review were identified. The selection process is illustrated in figure 1.

The majority of studies were from the USA, only three were from Europe, i.e., Austria [34], Portugal [35], and Sweden [36]. Six studies reported data on 173 adolescent patients before bariatric surgery with the number of patients ranging from 10 to 40 [34, 36-40]; six studies reported data on 227 adolescent patients after bariatric surgery with the number of patients ranging from 10 to 101 [35, 36, 41-45]. The gender in all studies was predominantly female, except for one [34]. BMI values ranged from 46.1 to $64.5 \mathrm{~kg} / \mathrm{m}^{2}$.

The study designs varied between retrospective reviews of clinical charts of patients [37-40,45], clinical follow-ups after examinations have been carried out [34, 41], and prospective feasibility, safety or observational studies [35, 36, 42-44]. Surgical procedures were either LAGB $[34,35,37,41-42]$ or RYGB $[36,39,43-45]$ or were not specified $[38,40]$. Most studies dealt with depressive disorders and depressive disorder symptoms in bariatric surgery patients, while one [36] described depressive and anxiety disorder symptoms from a pre- and post-operative point of view and another reported on pre-operative eating disorder symptoms [34]. The summarized characteristics of pre- and post-operative findings are presented in table 1 and 2, respectively. 
Herget et al.: Psychosocial Status and Mental Health in Adolescents before and after

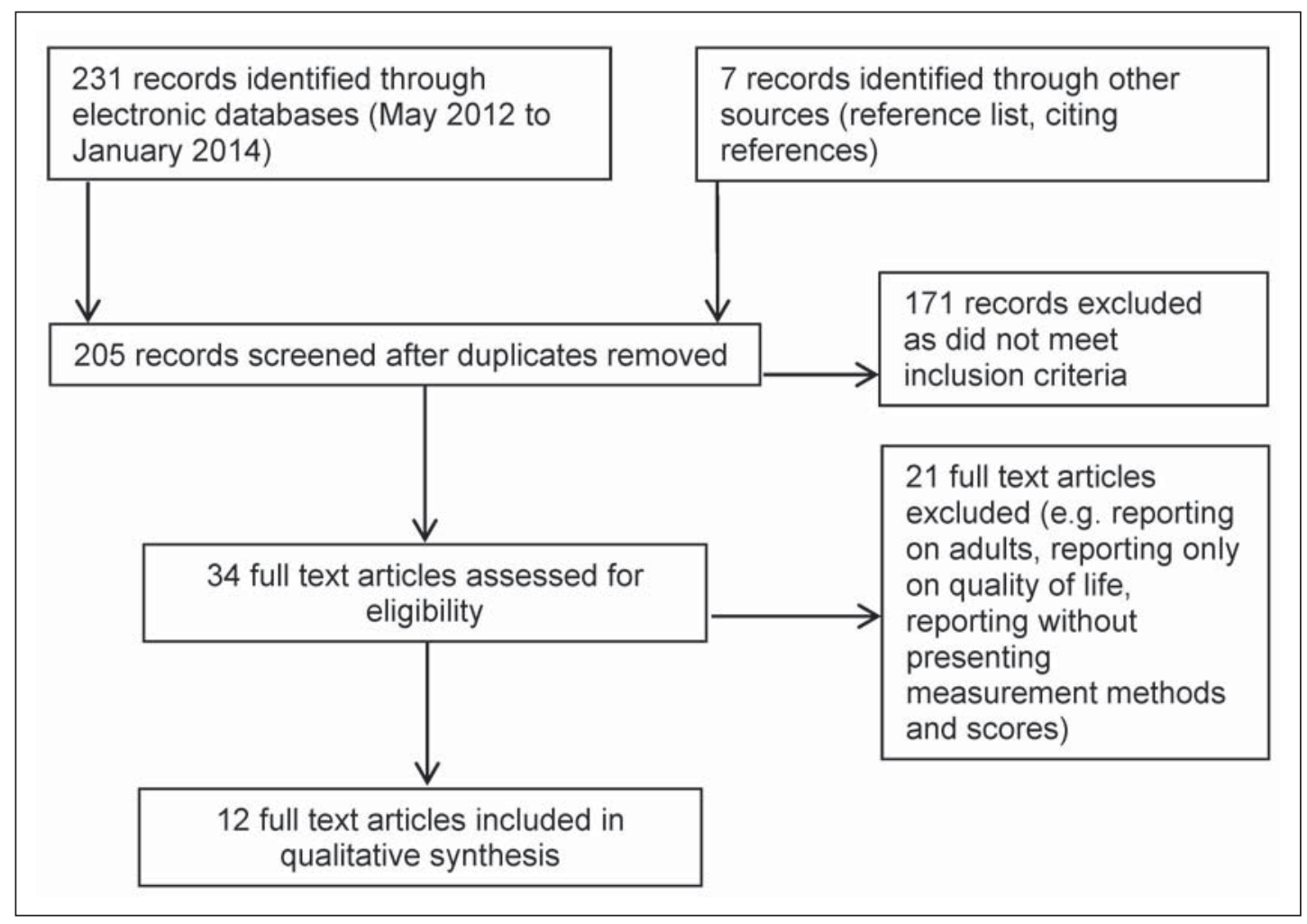

Fig. 1. Flowchart of literature search performed. Articles found and selected for review using PsychINFO, Pubmed and Medline electronic databases.

\section{Preoperative Mental Health of Adolescent Bariatric Surgery Candidates}

Six articles addressed mental health problems and behavioral disorders in adolescent bariatric surgery candidates. Depressive disorders, anxiety and eating disorders as well as respective symptoms were among the most common psychiatric phenomena documented.

\section{Depressive Disorders and Depressive Disorder Symptoms}

Four studies examined patients' baseline characteristics in relation to depressive disorders and depressive disorder symptoms by retrospectively analyzing clinical patient charts [37-40] (table 1). Medical information and psychosocial evaluation prior to surgery were used to assess adolescent mental health status. Only two studies reported on clinical interviews by mental health professionals [37, 38]; however, all four studies showed results of responses to the Beck Depression Inventory (BDI) [46] or its revised version (BDI-II) [47]. Both are quantitative self-report questionnaires with 21 questions assessing the severity of depressive symptoms on a 4-point Likert scale ranging from 0 to 3 . The sum score of the BDI-II indicates minimal (BDI $\leq 13)$, mild ( $14 \leq \mathrm{BDI} \leq 19)$, moderate $(20 \leq \mathrm{BDI} \leq 28)$, or severe $(29 \leq \mathrm{BDI} \leq 63)$ depressive symptoms. Clinically relevant depressive symptoms are commonly defined by moderate or severe scores.

From the two studies that also reported on clinical interviews, one did not specify diagnoses [37]. The other revealed that two thirds of the bariatric surgery candidates were clinically diagnosed with depressive disorders [38]. Similar results were found in studies using the BDI, reporting moderate and severe depressive disorder symptoms in up to $15 \%$ of the patients [40]. In another study, depressive disorder symptoms were assessed twice before bariatric surgery. Moderate and severe depressive disorder symptoms were reported in 
Herget et al.: Psychosocial Status and Mental Health in Adolescents before and after Bariatric Surgery: A Systematic Literature Review

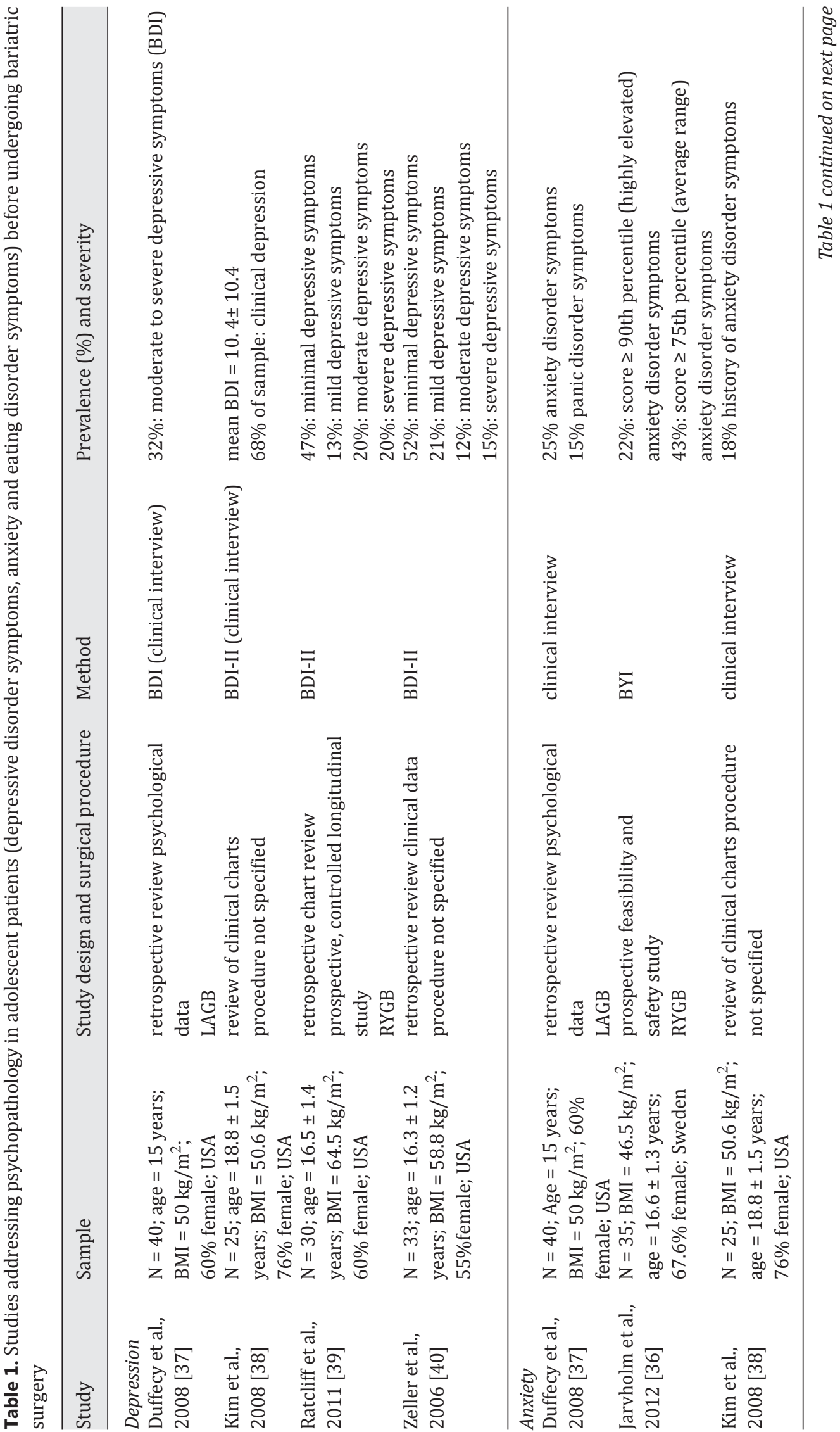



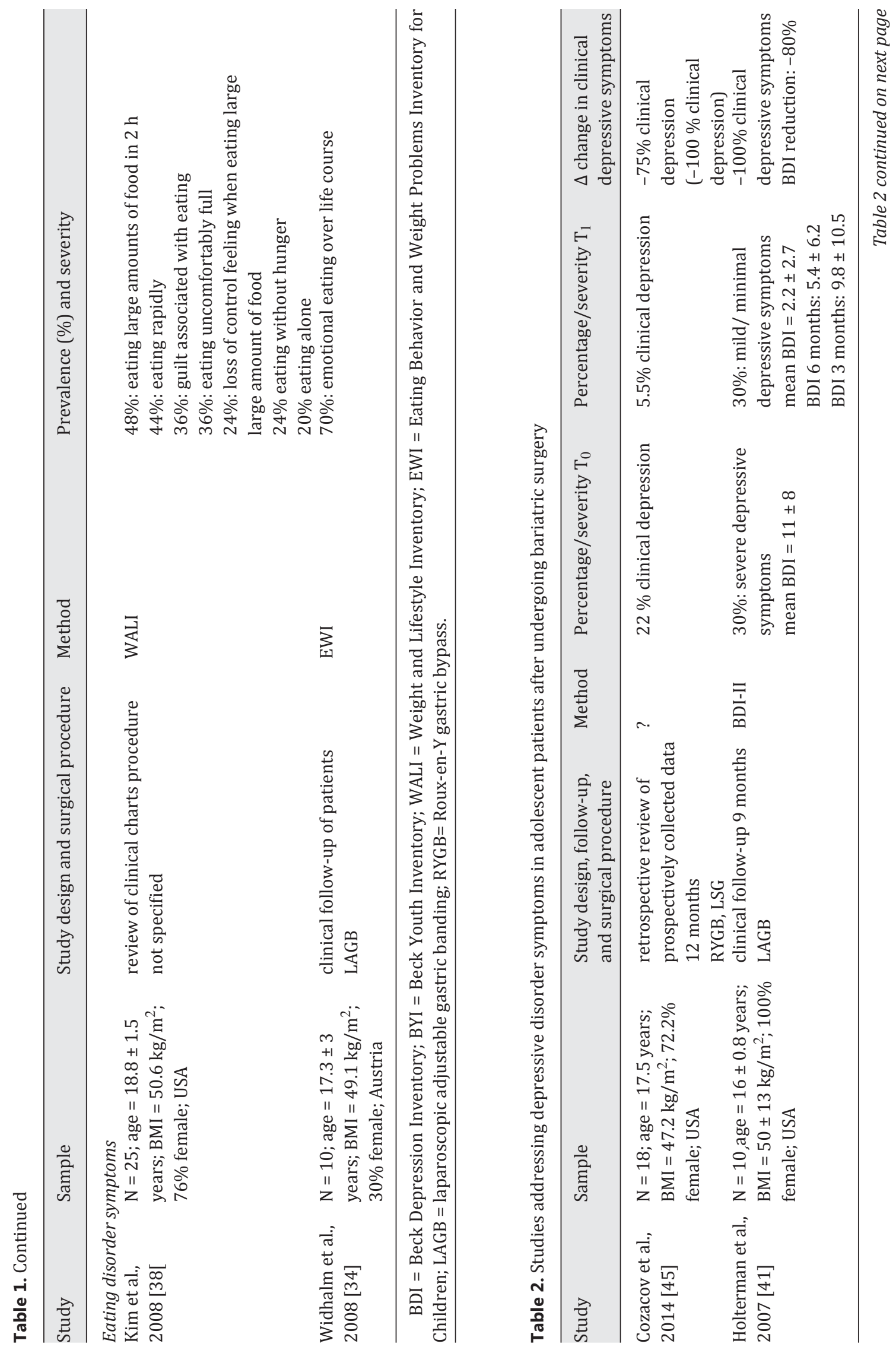
Herget et al.: Psychosocial Status and Mental Health in Adolescents before and after Bariatric Surgery: A Systematic Literature Review

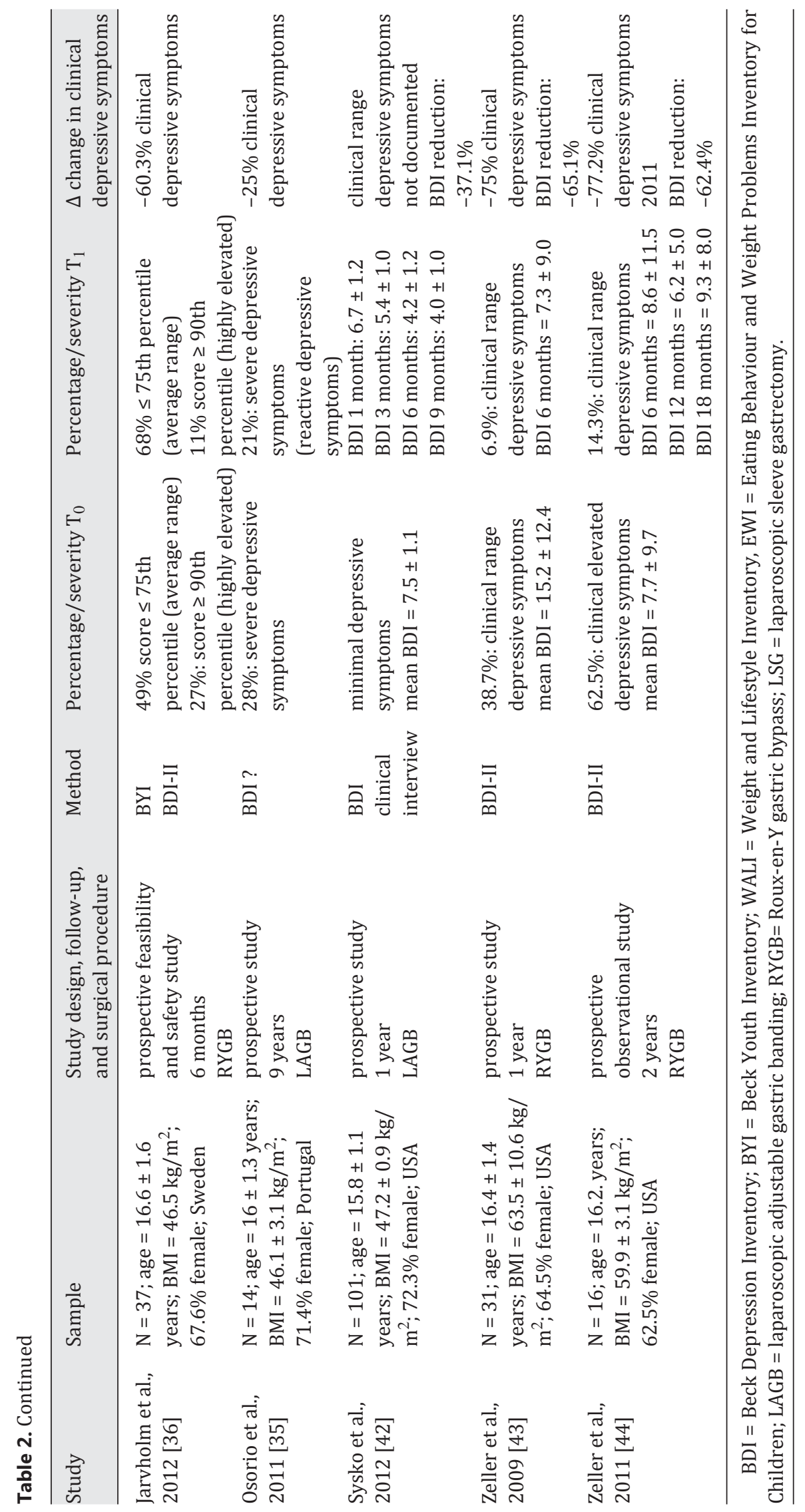


Herget et al.: Psychosocial Status and Mental Health in Adolescents before and after Bariatric Surgery: A Systematic Literature Review

almost half of the patients 6 months before surgery, and the rates remained stable immediately before surgery [39]. Information on the application of any antidepressant medication before surgery was not available in the studies included.

\section{Anxiety Disorders and Anxiety Disorder Symptoms}

Anxiety disorder symptoms have rarely been focused on when reporting patient characteristics before bariatric surgery. So far, only three studies reported rates of anxiety disorder symptoms in adolescent bariatric surgery candidates [36-38]; however, all used different methods. One study applied a validated clinical interview to diagnose a lifetime history of anxiety disorders [38], and another used a semi-structured clinical interview to report anxiety disorder symptoms rather than diagnoses [37]. In a third study, anxiety disorder symptoms were reported by using the Beck Youth Inventory (BYI) [36]. This questionnaire was developed to assess emotional and social impairment in childhood and adolescence with validated cut-off levels [48]. In summary, a lifetime history of anxiety disorders was found in $18 \%$ of bariatric surgery candidates, and anxiety disorder symptoms were found in $22 \%$ according to BYI reports and in 33\% of adolescents according to clinical interviews before bariatric surgery.

\section{Eating Disorders and Eating Disorder Symptoms}

Eating disorders (e.g., binge eating disorder) and eating disorder symptoms (e.g., loss of control eating) are both essential when assessing eligibility of bariatric surgery patients. Studies on adult patients show inconsistent findings regarding eating behavior pathology and weight loss surgery outcomes. Some studies reported that pre-operative eating disorders were not reliable predictors of excessive weight loss $[49,50]$. Other studies, however, claim that they might interfere with adherence to post-operative lifestyle recommendations [51]. So far, only two studies reported on pre-operative eating disorder symptoms in adolescent bariatric surgery patients $[34,38]$.

Both studies examined patients' life records for eating disorders and eating disorder symptoms retrospectively. In one study [38], patients did not meet criteria for any eating disorder; however, almost half of them either showed symptoms of loss of control eating or symptoms of binge eating disorder (e.g., eating unusually large amounts of food, experiencing guilt during the episode, eating until uncomfortably full and eating without being hungry). The other study found that $70 \%$ of adolescent patients reported eating disorder symptoms (e.g., emotional eating) [34].

\section{Combined Psychopathology}

The study of co-occurrence of mental disorders in adolescent patients has only been examined in one scientific report, which describes latent classes of psychopathology in detail [52]. $25 \%$ of all adolescent bariatric surgery candidates had depressive symptoms in the clinical range and a high probability for suffering from binge eating episodes (73.2-100\%), bulimic episodes (73.1\%), or night eating episodes (52.1\%).

\section{Effects of Bariatric Surgery on Post-Operative Mental Health Status}

Studies examining symptoms of anxiety and eating disorders in adolescents before and after bariatric surgery were not identified. Six studies reported on the course of depressive disorder symptoms in adolescent bariatric surgery patients [35, 36, 41-44] (table 2).

All studies reported on the use of either the BDI or the BDI-II, while only one study stated the use of a clinical interview [42]. Studies considerably differed with regard to the time point at which post-operative psychopathology was measured [36, 43, 44]. Use of antidepressants was not reported. 
Herget et al.: Psychosocial Status and Mental Health in Adolescents before and after Bariatric Surgery: A Systematic Literature Review

Six months and 9 months after surgery, improvements of mean levels of depressive symptoms were observed in all studies [36, 41-44]. In two studies, the lowest level of depressive symptoms was reported 1 year after surgery $[43,44]$. Another study documented improvement of clinical depression in $75 \%$ of all affected patients [45]. Tendencies towards an increased level of depressive disorder symptoms were observed 18 to 24 months after surgery [44]. One study, however, reported lowest levels of depressive disorder symptoms 9 months after bariatric surgery with an increase afterwards [42]. In general, prevalence of post-operative depressive disorders or depressive disorder symptoms was higher in female than in male adolescents [43]. Studies primarily reporting weight loss after bariatric surgery showed inconsistent findings on depressive symptoms of adolescent patients as secondary outcomes $[35,41]$. Nine months after LAGB, depressive disorder symptoms were completely resolved in one study [41].

In contrast, a long-term follow-up of 9 years after bariatric surgery revealed that $21.4 \%$ of all patients were still suffering from clinical depressive symptoms. However, in follow-up interviews most patients reported depressive symptoms due to other causes than obesity (e.g., death of a relative) [35, 45].

\section{Discussion}

The present study is a detailed systematic literature review and identified 12 studies that comprehensively focused on psychosocial status and mental health among adolescents before and after bariatric surgery.

Findings documented that adolescent bariatric surgery patients suffered from psychopathologies such as depressive disorders, anxiety disorders, and eating disorders. Before bariatric surgery, prevalence rates of depressive disorder symptoms ranged from $15 \%$ to almost $70 \%$, anxiety disorder symptoms were reported in $15-33 \%$ of the adolescents, and eating disorder symptoms were present among 48-70\%.

Severe depressive disorder symptoms in adolescents indicate the need for clinical attention and clearance before surgery. In comparison with recent studies that reported a major depressive disorder in $3.2 \%$ of all adolescents in the USA [53,54], the results of our review emphasize the high prevalence of depressive disorder symptoms in adolescent bariatric surgery patients. Similarly, mental health problems and disorders were found to be elevated among adult patients seeking bariatric surgery as compared with national surveys [55]. However, in contrast to adult bariatric surgery, no changes in levels of depressive disorder symptoms were reported from adolescents who were evaluated twice before bariatric surgery [39]. Socially desirable responding has been repeatedly found in adult patients, who indicated lower depressive symptoms in psychosocial assessments before bariatric surgery, termed as 'impression management' [56, 57].

Prevalence of anxiety was rarely systemically examined. As indicated by few studies, approximately one quarter of the adolescent bariatric surgery candidates seemed to suffer from anxiety disorder symptoms. Further research is warranted to examine type, severity, and duration of anxiety disorder symptoms and to delineate possible treatment options if this shows to be associated with reduced weight loss success after surgery. $18 \%$ of adult patients before bariatric surgery were diagnosed with a current anxiety disorder, such as generalized anxiety disorder $[58,59]$. Anxiety has been associated with low weight loss in adults [51].

Among the few available studies on eating disorder symptoms in adolescent bariatric surgery patients, the prevalence for binge eating according to self-reports ranged from 25 to $48 \%$. In adult bariatric surgery candidates, $10-16 \%$ of patients suffered from binge eating disorder $[60,61]$. Combined psychopathology (depressive, anxiety and eating disorder 
Herget et al.: Psychosocial Status and Mental Health in Adolescents before and after Bariatric Surgery: A Systematic Literature Review

symptoms) was only reported in one study [52] and needs further attention to delineate risk profiles of adolescent bariatric surgery patients.

With regard to post-operative psychosocial status and mental health, the present review documents positive effects of levels of depressive disorder symptoms associated with adolescent bariatric surgery. According to the studies reviewed, depressive disorder symptoms either remitted or improved after bariatric surgery. Overall, depressive symptoms tended to decrease towards a milder symptomatology after the first 12 months following surgery. However, some evidence exists from adult bariatric surgery populations that persisting or re-occurring psychopathology regularly becomes apparent at the end of the post-operative 'honeymoon' phase following surgery $[62,63]$. Furthermore, in adolescents correlations between weight loss success and improvement of depressive disorder symptoms were not reported as they have been in adults before [59].

This review was limited by several methodological issues in the studies themselves. First, sample sizes were small and ranged from 10 to a hundred patients. Thus, larger sample sizes are necessary to document accurate prevalence rates of psychopathology. Furthermore, sociodemographic and ethnic characteristics as well as family background of patients were not examined in detail and need to be further elucidated.

Second, pre- and post-operative assessments of depressive, anxiety and eating disorder symptoms were inconsistent in the studies reviewed. Standardized clinical interviews with adolescent surgery candidates were only reported in some studies [37, 38], and, if applied, cut-off points for the definition of clinically relevant depressive disorder symptoms varied. For better comparability of the studies and their outcomes, documentation of consistent cut-off values is inevitable. Also, four studies were retrospective chart reviews and are limited regarding their explanatory power of severity and causes for mental health disorders. According to the current bariatric surgery guidelines, a comprehensive assessment should incorporate documentation of both standardized questionnaires and clinical interviews [64]. Additionally, suicidal ideation, substance abuse, and impulse control disorders have not yet been examined in the adolescent population although they were documented in adult bariatric surgery patients $[55,60,65]$.

Our review has pointed out that depressive disorder symptoms are likely to be present in adolescents and that pathological eating patterns (e.g., loss of control eating) also emerged after bariatric surgery in adolescents. Among adult bariatric surgery patients, post-operative depressive disorders and eating disorder symptoms were associated with less favorable post-operative weight loss outcome $[59,66]$. Thus, assessment may improve the choice of patients suited for surgery, while treatment of pre- and post-operative depressive and eating disorder symptoms can have an impact on successful weight loss after surgery. As has been shown recently, post-operative behavioral management has a positive effect on weight loss among adult bariatric surgery patients [66].

The effect of psychopathology changes before and after bariatric surgery on outcomes of adolescent bariatric surgery has rarely been studied. Pre-operative depressive disorder symptoms neither predicted initial BMI nor rate of BMI change after bariatric surgery [42]. Previously, associations between depressive disorder symptoms and poor adherence to conservative obesity treatment in adolescents were documented [67]. To further analyze this association, more studies are needed focusing on long-term follow-up of surgery outcomes and their correlation with development of psychopathology over the course of weight loss treatment of adolescents.

In conclusion, our review highlights the prevalence and severity of mental health disorders before and after bariatric surgery. Future research needs to focus on establishing valid and standardized clinical assessment procedures of psychopathology in adolescent bariatric surgery patients. By further examining the mental health status of the adolescent 
bariatric surgery patients and its effect on long-term outcomes of bariatric surgery, the safety and effectiveness of bariatric surgery programs for adolescents as well as pre- and post-operative care can be improved.

\section{Acknowledgement}

The authors of this paper would like to acknowledge the contribution of David Petroff, PhD, University of Leipzig, for proof-reading the manuscript. This work was supported by the Federal Ministry of Education and Research, Germany (Integrated Research and Treatment Center IFB Adiposity Diseases, FKZ: 01E01001).

\section{Disclosure Statement}

The authors declare that they have no conflicts of interests.

\section{References}

1 World Health Organization: Obesity and overweight; in WHO (ed): Fact Sheet. Geneva, WHO, 2012.

2 Cole TJ, Bellizzi MC, Flegal KM, Dietz WH: Establishing a standard definition for child overweight and obesity worldwide: international survey. BMJ 2000;320:1240-1243.

3 Rolland-Cachera MF: Childhood obesity: current definitions and recommendations for their use. Int J Pediatr Obes 2011;6:325-331.

4 Olds T, Maher C, Zumin S, Peneau S, Lioret S, Castetbon K, Bellisle, de Wilde J, Hohepa M, Maddison R, Lissner L, Sjoberg A, Zimmermann M, Aeberli I, Ogden C, Flegal K, Summerbell C: Evidence that the prevalence of childhood overweight is plateauing: data from nine countries. Int J Pediatr Obes 2011;6:342-360.

5 Bluher S, Meigen C, Gausche R, Keller E, Pfaffle R, Sabin M, Werther G, Odeh R, Kiess W: Age-specific stabilization in obesity prevalence in German children: a cross-sectional study from 1999 to 2008. Int J Pediatr Obes 2011;6:e199-206.

6 Kalarchian MA, Marcus MD: Psychiatric comorbidity of childhood obesity. Int Rev Psychiatry 2012;24:241246.

7 Lavie CJ, Milani RV, Ventura HO: Obesity and cardiovascular disease: risk factor, paradox, and impact of weight loss. J Am Coll Cardiol 2009;53:1925-1932.

8 Kalarchian MA, Marcus MD: Preoperative weight loss in bariatric surgery. Obes Surg 2009;19:539.

9 Oude Luttikhuis H, Baur L, Jansen H, Shrewsbury VA, O’Malley C, Stolk RP, Summerbell CD. Interventions for treating obesity in children. Cochrane Database Syst Rev. 2009:CD001872.

10 Oyetunji TA, Franklin AL, Ortega G, Akolkar N, Qureshi FG, Abdullah F, Cornwell EE, Nwomeh BC, Fullum TM: Revisiting childhood obesity: persistent underutilization of surgical intervention? Am Surg 2012;78:788-793.

11 Sarwer DB, Dilks RJ: Invited commentary: childhood and adolescent obesity: psychological and behavioral issues in weight loss treatment. J Youth Adolesc 2012;41:98-104.

12 Bondada S, Jen HC, Deugarte DA: Outcomes of bariatric surgery in adolescents. Curr Opin Pediatr 2011;23: 552-556.

13 Kaul A, Sharma J: Impact of bariatric surgery on comorbidities. Surg Clin North Am 2011;91:1295-1312, ix.

14 Terranova L, Busetto L, Vestri A, Zappa MA: Bariatric surgery: cost-effectiveness and budget impact. Obes Surg 2012;22:646-653.

15 Hsia DS, Fallon SC, Brandt ML: Adolescent bariatric surgery. Arch Pediatr Adolesc Med 2012;166:757-766.

16 Alqahtani AR, Antonisamy B, Alamri H, Elahmedi M, Zimmerman VA: Laparoscopic sleeve gastrectomy in 108 obese children and adolescents aged 5 to 21 years. Ann Surg 2012;256:266-273.

17 Barnett SJ: Contemporary surgical management of the obese adolescent. Curr Opin Pediatr 2011;23:351-355.

18 Inge TH, Xanthakos SA, Zeller MH: Bariatric surgery for pediatric extreme obesity: now or later? Int J Obes (Lond) 2007;31:1-14.

19 Livingston EH: Surgical treatment of obesity in adolescence. JAMA 2010;303:559-560.

20 Pallati P, Buettner S, Simorov A, Meyer A, Shaligram A, Oleynikov D: Trends in adolescent bariatric surgery evaluated by UHC database collection. Surg Endosc 2012;26:3077-3081.

21 Treadwell JR, Sun F, Schoelles K: Systematic review and meta-analysis of bariatric surgery for pediatric obesity. Ann Surg 2008;248:763-776.

22 Sebastian C, Burnett S, Blakemore SJ: Development of the self-concept during adolescence. Trends Cogn Sci 2008;12:441-446.

23 Vander Wal JS: The relationship between body mass index and unhealthy weight control behaviors among adolescents: the role of family and peer social support. Econ Hum Biol 2012;10:395-404. 
Herget et al.: Psychosocial Status and Mental Health in Adolescents before and after

Bariatric Surgery: A Systematic Literature Review

24 Puder JJ, Munsch S: Psychological correlates of childhood obesity. Int J Obes (Lond) 2010;34(suppl 2):S37-43.

25 Goldfield GS, Moore C, Henderson K, Buchholz A, Obeid N, Flament MF: Body dissatisfaction, dietary restraint, depression, and weight status in adolescents. J Sch Health 2010;80:186-192.

26 Bocchieri-Ricciardi LE, Chen EY, Munoz D, Fischer S, Dymek-Valentine M, Alverdy JC, le Grange D: Pre-surgery binge eating status: effect on eating behavior and weight outcome after gastric bypass. Obes Surg 2006;16: 1198-1204.

27 van Hout GC, Hagendoren CA, Verschure SK, van Heck GL: Psychosocial predictors of success after vertical banded gastroplasty. Obes Surg 2009;19:701-707.

28 Kudsi OY, Huskey K, Grove S, Blackburn G, Jones DB, Wee CC: Prevalence of preoperative alcohol abuse among patients seeking weight-loss surgery. Surg Endosc 2013;27:1093-1097.

29 Livhits M, Mercado C, Yermilov I, Parikh JA, Dutson E, Mehran A, Ko CY, Gibbons MM: Preoperative predictors of weight loss following bariatric surgery: systematic review. Obes Surg 2012;22:70-89.

30 Pull CB: Current psychological assessment practices in obesity surgery programs: what to assess and why. Curr Opin Psychiatry 2010;23:30-36.

31 Sysko R, Zandberg LJ, Devlin MJ, Annunziato RA, Zitsman JL, Walsh BT: Mental health evaluations for adolescents prior to bariatric surgery: a review of existing practices and a specific example of assessment procedures. Clin Obes 2013;3:62-72.

32 Kubik JF, Gill RS, Laffin M, Karmali S: The impact of bariatric surgery on psychological health. J Obes 2013; 2013:837989.

33 Nadler EP, Brotman LM, Miyoshi T, Fryer GE Jr, Weitzman M: Morbidity in obese adolescents who meet the adult National Institutes of Health criteria for bariatric surgery. J Pediatr Surg 2009;44:1869-1876.

34 Widhalm K, Dietrich S, Prager G, Silberhummer G, Orth D, Kispal ZF: Bariatric surgery in morbidly obese adolescents: a 4-year follow-up of ten patients. Int J Pediatr Obes 2008;3(suppl 1):78-82.

35 Osorio A, Moreira-Pinto J, Pereira J, Silva G, Bonet B, Cidade-Rodrigues JA, Ferreira-de-Sousa JA, Enes C, Mansilha H: 9 years after the first laparoscopic adjusted gastric banding (LAGB) in adolescents: the Portuguese experience. Zeitschr Kinderchir 2011;21:331-334.

36 Jarvholm K, Olbers T, Marcus C, Marild S, Gronowitz E, Friberg P, Johnsson P, Flodmark CE: Short-term psychological outcomes in severely obese adolescents after bariatric surgery. Obesity (Silver Spring) 2012;20:318323.

37 Duffecy J, Bleil ME, Labott SM, Browne A, Galvani C: Psychopathology in adolescents presenting for laparoscopic banding. J Adolesc Health 2008;43:623-625.

38 Kim RJ, Langer JM, Baker AW, Filter DE, Williams NN, Sarwer DB: Psychosocial status in adolescents undergoing bariatric surgery. Obes Surg 2008;18:27-33.

39 Ratcliff MB, Reiter-Purtill J, Inge TH, Zeller MH: Changes in depressive symptoms among adolescent bariatric candidates from preoperative psychological evaluation to immediately before surgery. Surg Obes Relat Dis 2011;7:50-54.

40 Zeller MH, Roehrig HR, Modi AC, Daniels SR, Inge TH: Health-related quality of life and depressive symptoms in adolescents with extreme obesity presenting for bariatric surgery. Pediatrics 2006;117:1155-1161.

41 Holterman AX, Browne A, Dillard BE 3rd, Tussing L, Gorodner V, Stahl C, Browne N, Labott S, Herdegen J, Guzman G, Rink A, Nwaffo I, Galvani C, Horgan S, Holterman M: Short-term outcome in the first 10 morbidly obese adolescent patients in the FDA-approved trial for laparoscopic adjustable gastric banding. J Pediatr Gastroenterol Nutr 2007;45:465-473.

42 Sysko R, Devlin MJ, Hildebrandt TB, Brewer SK, Zitsman JL, Walsh BT: Psychological outcomes and predictors of initial weight loss outcomes among severely obese adolescents receiving laparoscopic adjustable gastric banding. J Clin Psychiatry 2012;73:1351-1357.

43 Zeller MH, Modi AC, Noll JG, Long JD, Inge TH: Psychosocial functioning improves following adolescent bariatric surgery. Obesity (Silver Spring) 2009;17:985-990.

44 Zeller MH, Reiter-Purtill J, Ratcliff MB, Inge TH, Noll JG: Two-year trends in psychosocial functioning after adolescent Roux-en-Y gastric bypass. Surg Obes Relat Dis 2011;7:727-732.

45 Cozacov Y, Roy M, Moon S, Marin P, Menzo EL, Szomstein S, Rosenthal R: Mid-term results of laparoscopic sleeve gastrectomy and Roux-en-Y gastric bypass in adolescent patients. Obes Surg 2014;24:747-752.

46 Beck AT, Ward CH, Mendelson M, Mock J, Erbaugh J: An inventory for measuring depression. Arch Gen Psychiatry 1961;4:561-571.

47 Beck AT, Steer RA, Ball R, Ranieri W: Comparison of Beck Depression Inventories -IA and -II in psychiatric outpatients. J Pers Assess 1996;67:588-597.

48 Beck J, Beck A, Jolly J: Beck Youth Inventories of Emotional and Social Impairment Manual. San Antonio, Psychological Corporation, 2001.

49 Facchiano E, Scaringi S, Quartararo G, Alpigiano G, Liscia G, Pavoni V, Lucchese M: Do preoperative eating behaviors influence weight loss after biliopancreatic diversion? Obes Surg 2013;23:2080-2085.

50 Wood KV, Ogden J: Explaining the role of binge eating behaviour in weight loss post bariatric surgery. Appetite 2012;59:177-180.

51 Brunault P, Jacobi D, Miknius V, Bourbao-Tournois C, Huten N, Gaillard P, Couet C, Camus V, Ballon N: High preoperative depression, phobic anxiety, and binge eating scores and low medium-term weight loss in sleeve gastrectomy obese patients: a preliminary cohort study. Psychosomatics 2012;53:363-370. 
Herget et al.: Psychosocial Status and Mental Health in Adolescents before and after

Bariatric Surgery: A Systematic Literature Review

52 Sysko R, Zakarin EB, Devlin MJ, Bush J, Walsh BT: A latent class analysis of psychiatric symptoms among 125 adolescents in a bariatric surgery program. Int J Pediatr Obes 2011;6:289-297.

53 Faith MS, Butryn M, Wadden TA, Fabricatore A, Nguyen AM, Heymsfield SB: Evidence for prospective associations among depression and obesity in population-based studies. Obes Rev 2011;12:e438-453.

54 Merikangas AK, Mendola P, Pastor PN, Reuben CA, Cleary SD: The association between major depressive disorder and obesity in US adolescents: results from the 2001-2004 National Health and Nutrition Examination Survey. J Behav Med 2012;35:149-154.

55 Mitchell JE, Selzer F, Kalarchian MA, Devlin MJ, Strain GW, Elder KA, Marcus MD, Wonderlich S, Christian NJ, Yanovski SZ: Psychopathology before surgery in the longitudinal assessment of bariatric surgery-3 (LABS-3) psychosocial study. Surg Obes Relat Dis 2012;8:533-541.

56 Ambwani S, Boeka AG, Brown JD, Byrne TK, Budak AR, Sarwer DB, Fabricatore AN, Morey LC, O'Neil PM: Socially desirable responding by bariatric surgery candidates during psychological assessment. Surg Obes Relat Dis 2013;9:300-305.

57 Fabricatore AN, Sarwer DB, Wadden TA, Combs CJ, Krasucki JL: Impression management or real change? Reports of depressive symptoms before and after the preoperative psychological evaluation for bariatric surgery. Obes Surg 2007;17:1213-1219.

58 de Zwaan M, Enderle J, Wagner S, Muhlhans B, Ditzen B, Gefeller O, Mitchell JE, Muller A: Anxiety and depression in bariatric surgery patients: a prospective, follow-up study using structured clinical interviews. J Affect Disord 2011;133:61-68.

59 Kalarchian MA, Marcus MD, Levine MD, Courcoulas AP, Pilkonis PA, Ringham RM, Soulakova JN, Weissfeld LA, Rofey DL: Psychiatric disorders among bariatric surgery candidates: relationship to obesity and functional health status. Am J Psychiatry 2007;164:328-334; quiz 374.

60 Muller A, Claes L, Mitchell JE, Fischer J, Horbach T, de Zwaan M: Binge eating and temperament in morbidly obese prebariatric surgery patients. Eur Eat Disord Rev 2012;20:e91-95.

61 White MA, Kalarchian MA, Masheb RM, Marcus MD, Grilo CM: Loss of control over eating predicts outcomes in bariatric surgery patients: a prospective, 24-month follow-up study. J Clin Psychiatry 2010;71:175-184.

62 Bocchieri LE, Meana M, Fisher BL: A review of psychosocial outcomes of surgery for morbid obesity. J Psychosom Res 2002;52:155-165.

63 van Hout GC, Boekestein P, Fortuin FA, Pelle AJ, van Heck GL: Psychosocial functioning following bariatric surgery. Obes Surg 2006;16:787-794.

64 Michalsky M, Kramer RE, Fullmer MA, Polfuss M, Porter R, Ward-Begnoche W, Getzoff EA, Dreyer M, Stolzman S, Reichard KW: Developing criteria for pediatric/adolescent bariatric surgery programs. Pediatrics 2011; 128(suppl 2):S65-70.

65 Peterhansel C, Petroff D, Klinitzke G, Kersting A, Wagner B: Risk of completed suicide after bariatric surgery: a systematic review. Obes Rev 2013;14:369-382.

66 Rudolph A, Hilbert A: Post-operative behavioural management in bariatric surgery: a systematic review and meta-analysis of randomized controlled trials. Obes Rev 2013;14:292-302.

67 Zeller M, Kirk S, Claytor R, Khoury P, Grieme J, Santangelo M, Daniels S: Predictors of attrition from a pediatric weight management program. J Pediatr 2004;144:466-470. 